15

\title{
Эксперимент Нейтрино-4 по поиску стерильного нейтрино с многосекционной моделью детектора
}

\author{
(c) А.П. Серебров, ${ }^{1}$ В.Г. Ивочкин, ${ }^{1}$ Р.М. Самойлов, ${ }_{1}^{1}$ А.К. Фомин, ${ }^{1}$ В.Г. Зиновьев, ${ }_{1}^{1}$ \\ П.В. Неустроев, ${ }^{1}$ В.Л. Головцов, ${ }^{1}$ А.В. Чёрный, ${ }^{1}$ О.М. Жеребцов, ${ }^{1}$ А.О. Полюшкин, ${ }^{1}$ \\ В.П. Мартемьянов, ${ }^{2}$ В.Г. Тарасенков, ${ }^{2}$ В.И. Алёшин, ${ }^{2}$ А.Л. Петелин, ${ }^{3}$ А.Л. Ижутов, ${ }^{3}$ \\ А.А. Тузов, ${ }^{3}$ С.А. Сазонтов, ${ }^{3}$ Д.К. Рязанов, ${ }^{3}$ М.О. Громов, ${ }^{3}$ \\ B.В. Асранасьев, ${ }^{3}$ M.E. Зайцев, ${ }^{1}$ М.Е. Чайковский ${ }^{1}$
}

${ }^{1}$ Петербургский институт ядерной фризики им. Б.П. Константинова, Национальный исследовательский центр „Курчатовский институт“, 188300 Гатчина, Ленинградская область, Россия

${ }^{2}$ Национальный исследовательский центр „Курчатовский институт“, 123182 Москва, Россия

${ }^{3}$ ОАО „ГНЦ НИИАР",

433510 Димитровград, Россия

e-mail: serebrov@pnpi.spb.ru

(Поступило в Редакцию 20 июня 2016 г.)

В связи с вопросом о возможном существовании стерильного нейтрино, на базе реактора СМ-3 (Димитровград, Россия) была создана лаборатория для поиска осцилляций реакторных антинейтрино. В середине 2015 г. был установлен прототип многосекционного нейтринного детектора с объемом жидкого сцинтиллятора 3501. Этот детектор может перемещаться внутри пассивной защиты в диапазоне расстояний от 6 до $11 \mathrm{~m}$ от активной зоны реактора. Впервые выполнены измерения зависимости потока антинейтрино на столь близких расстояниях от активной зоны реактора с перемещаемым детектором. Измерения с прототипом многосекционного детектора показали, что в сложных условиях космического фона на поверхности Земли можно проводить измерения потока антинейтрино от реактора.

DOI: 10.21883/JTF.2017.02.44141.1940

\section{Введение}

В настоящее время широко обсуждается вопрос о возможном существовании стерильного нейтрино, имеющего намного меньшее сечение взаимодействия с веществом по сравнению, например, с реакторным электронным антинейтрино. Предполагается, что благодаря переходам реакторных антинейтрино в стерильное состояние возможно наблюдать эффект нейтринных осцилляций на малых расстояниях от реактора и дефицит потока антинейтрино на больших расстояниях [1,2]. Более того, стерильное нейтрино рассматривается как возможный кандидат на темную материю.

Идея осцилляций может быть проверена прямыми измерениями эффекта вариации потока и спектра антинейтрино на малых расстояниях от реактора. Детектор должен перемещаться, а также быть спектральночувствительным. В нашем эксперименте ставится задача подтвердить или опровергнуть на определенном уровне точности возможность существования стерильного нейтрино. Для поиска осцилляций в стерильном нейтрино необходимо зарегистрировать вариацию потока реакторных антинейтрино. Если такой процесс существует, то он может описываться уравнением осцилляций

$$
P\left(\tilde{v}_{e} \rightarrow \tilde{v}_{e}\right)=1-\sin ^{2} 2 \theta_{14} \sin ^{2}\left(1.27 \frac{\Delta m_{14}^{2}\left[\mathrm{eV}^{2}\right] L[\mathrm{~m}]}{E_{\tilde{v}}[\mathrm{MeV}]}\right),
$$

где $E_{\tilde{v}}$ есть энергия антинейтрино, а $\Delta m_{14}^{2}$ и $\sin ^{2} 2 \theta_{14}$ являются неизвестными параметрами процесса осцилляций.

Для осуществления эксперимента необходимо провести измерения потока антинейтрино и спектра на малых расстояниях, например 6-12 m от практически точечного источника антинейтрино.

Мы изучили возможность проведения новых экспериментов на российских исследовательских реакторах. Реактор СМ-3 в силу конструкционных особенностей предоставляет наилучшие условия для проведения экспериментов по поиску нейтринных осцилляций на малых расстояниях. Преимущества реактора СМ-3: компактная активная зона реактора $(35 \times 42 \times 42 \mathrm{~cm})$ при мощности $100 \mathrm{MW}$ и малое расстояние $(5 \mathrm{~m})$ от активной зоны до стены экспериментального зала. Кроме того, особую важность имеет тот факт, что поток нейтрино может измеряться в довольно широком диапазоне от 6 до $13 \mathrm{~m}[3,4]$. К сожалению, реактор находится на поверхности Земли, и космический фон составляет основную проблему эксперимента. Пассивная защита от космического излучения благодаря бетонным перекрытиям здания оценивается в 5-10 m водного эквивалента. В настоящей работе представлены исследования подавления фона с многосекционной моделью детектора и результаты первых измерений зависимости потока реакторных антинейтрино от расстояния от активной зоны реактора в диапазоне 6-11 m. 


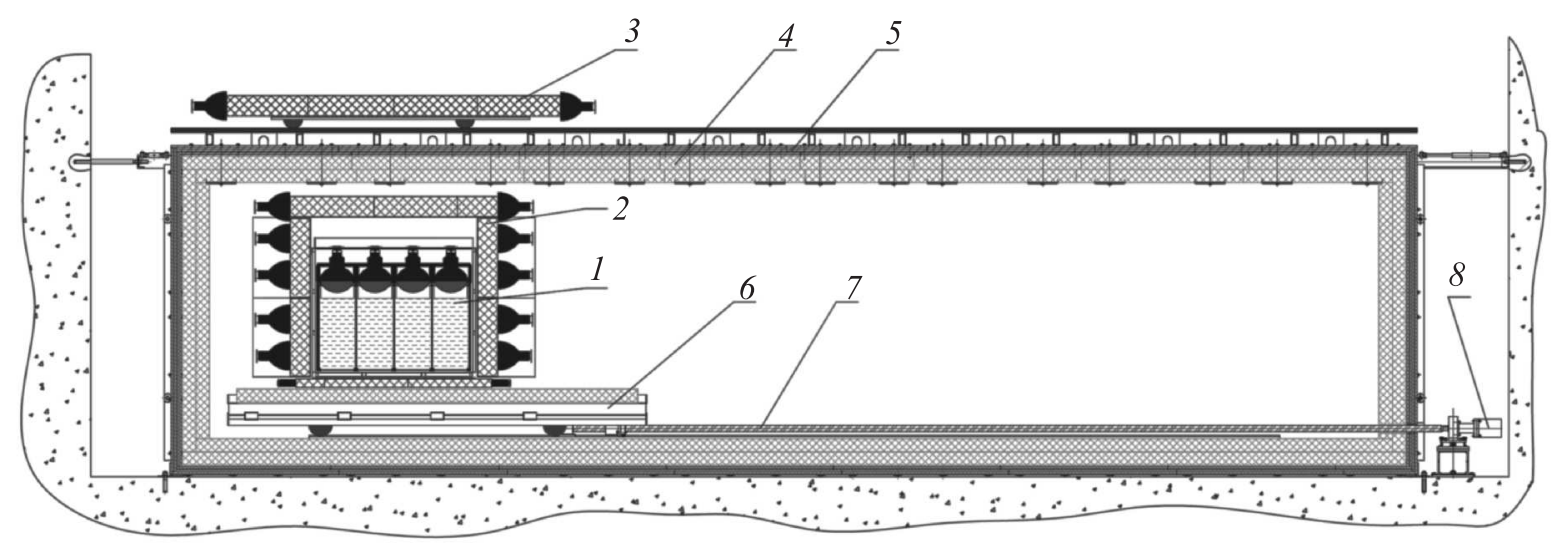

Рис. 1. Общая схема установки. 1 - детектор реакторных антинейтрино, 2 - внутренняя активная защита детектора, 3 - внешняя активная защита детектора (зонтик), 4 - пассивная защита детектора из железа и свинца, 5 - пассивная защита детектора из борированного полиэтилена, 6 - перемещаемая платформа, 7 - ходовой винт, 8 - шаговый двигатель.

\section{Проблема регистрации антинейтрино от реактора в условиях космического фона на поверхности Земли}

В нашем эксперименте были исследованы две модели детектора: первая модель с объемом жидкого сцинтиллятора 4001 на базе фотоумножителей ФЭУ-49Б без секционирования детектора и вторая модель на базе фотоумножителей ФЭУ-9354 с секционированием объема 3501 на 16 ячеек.

Детектор заполнен жидким сцинтиллятором с примесью $0.1 \% \mathrm{Gd}$. Работа детектора основана на реакции $\tilde{v}_{e}+p \rightarrow e^{+}+n$. Вначале происходит регистрация позитрона, энергия которого определяется энергией исходного антинейтрино, и регистрация двух гамма-квантов с энергией $511 \mathrm{keV}$ каждый. Вторичный сигнал регистрируется при захвате нейтрона гадолинием с образованием каскада гамма-квантов суммарной энергией около $8 \mathrm{MeV}$. Детекторная система записывает данные о двух последовательных сигналах от позитрона и нейтрона так называемые коррелированные сигналы.

Наиболее интенсивная загрузка детектора происходит от космического излучения и гамма квантов от окружающих материалов. Скорость счета детектора составляет приблизительно $10^{2} \mathrm{~s}^{-1}$. В условиях такой загрузки нужно выделить сигнал от реакторных антинейтрино, который составляет приблизительно $2 \cdot 10^{-3} \mathrm{~s}^{-1}$, т.е. меньший на 5 порядков величины. Применяя метод задержанных совпадений во временном интервале $100 \mu \mathrm{s}$, удается выделить коррелированные события с загрузкой приблизительно $1 \mathrm{~s}^{-1}$. Основная часть этих событий мюоны, остановившиеся в детекторе и захваченные ядрами с испарением нейтронов. Дискриминируя эти события по энергии, удается снизиться до уровня скорости счета коррелированных событий $0.1 \mathrm{~s}^{-1}$ [5]. За счет применения активной защиты детектора удается подавить фон еще почти на порядок величины. К сожалению, соотношение сигнал-фон остается на уровне $20-25 \%$, что существенно влияет на точность измерений потока антинейтрино от реактора. В связи с этим, кроме активной защиты детектора, были исследованы различные методы подавления фона: отбор сигналов по форме импульса и секционирование детектора.

\section{Внешняя и внутренняя активная защита детектора}

Активная защита нейтринного детектора состоит из двух уровней: внешнего и внутреннего (рис. 1). На крыше пассивной защиты над детектором установлена система активной защиты „зонтик“, состоящая из сцинтилляционных пластин площадью $0.5 \times 0.5 \mathrm{~m}$ и толщиной $12 \mathrm{~cm}$. Полная площадь „зонтика“ составляет $2 \times 3 \mathrm{~m}$. Принимая во внимание тот факт, что площадь детектора $0.9 \times 0.9 \mathrm{~m}$, такая защита должна захватывать основной поток мюонов, направленных на детектор. Однако этого недостаточно, и поэтому применен следующий уровень защиты - активная защита вокруг детектора. Она также состоит из сцинтилляционных пластин толщиной $12 \mathrm{~cm}$ и фотоумножителей ФЭУ-49Б. Нижняя плоскость внутренней активной защиты состоит из сцинтилляционных пластин толщиной $5 \mathrm{~cm}$ с фотоумножителями ФЭУ-97. На рис. 1 представлена схема установки.

Пассивная защита детектора состоит из элементов, построенных на основе стальных пластин $1 \times 2 \mathrm{~m}$ толщиной $10 \mathrm{~mm}$. К пластине присоединено 6 листов свинца толщиной $10 \mathrm{~mm}$. Объем внутренней камеры активной защиты составляет $2 \times 2 \times 8 \mathrm{~m}$. Изнутри камера покрыта пластинами борированного полиэтилена толщиной $16 \mathrm{~cm}$. Полная масса пассивной защиты составляет 60 тонн, объем борированного полиэтилена $10 \mathrm{~m}^{3}$. Внутри пассивной защиты на рельсах установлена платформа с детектором антинейтрино. Платформа приводится в движение шаговым двигателем и может быть установлена на расстоянии от 6 до $11 \mathrm{~m}$ от активной зоны реактора. 
Эффект подавления фона (реактор выключен) благодаря внешней активной защите составил в среднем по всему диапазону расстояний приблизительно 1.85 раза. При включенной внешней защите эффект дополнительного подавления фона с внутренней активной защитой составил $\sim 2.0$ раза. Полный эффект подавления двухуровневой активной защиты составил $\sim 3.7$ раза.

К сожалению, отношение сигнал-фон (в среднем по расстоянию) составляет $\sim 20 \%$. Здесь сигнал это разность между числом коррелированных событий при включенном и выключенном реакторе, а фон это число коррелированных событий при выключенном реакторе. При отношении сигнал-фон $20 \%$ точность измерений падает приблизительно в 3 раза по отношению к бесфоновым условиям. Поэтому требуется внедрение дополнительных методов подавления фона.

Есть как минимум два подхода к решению этого вопроса: использовать специфические особенности детектирования или позитрона, или протона отдачи. Для первого можно использовать то, что позитрон обязательно аннигилирует, и гамма-кванты разлетаются в противоположных направлениях. Для второго есть известный экспериментальный факт различия формы сигналов от тяжелых и легких частиц.

Из проведенных исследований было установлено, что разделение по форме импульса с данным сцинтиллятором работает только, когда фотоумножитель просматривает небольшую глубину, т.е. когда излучение, которое отвечает за медленную компоненту, достигает ФЭУ.

В связи с неудачей использования метода разделения сигналов от позитронов и протонов отдачи по форме импульса пришлось вернуться к рассмотрению другой возможности - того, что позитрон обязательно аннигилирует и появившиеся 2 гамма-кванта разлетятся в противоположных направлениях, т.е. к попытке детектирования гамма-квантов с энергией $511 \mathrm{keV}$. C этой целью была рассмотрена возможность создания детектора сотового типа.

\section{Модель детектора антинейтрино сотового типа}

Создание многосекционной системы имело целью детектировать появление позитрона в процессе нейтринной реакции. Как уже отмечалось, основную проблему эксперимента на поверхности Земли составляют быстрые нейтроны, возникающие от космического излучения. Процесс рассеяния быстрых нейтронов легко имитирует нейтринный процесс. Регистрация первого (стартового или мгновенного сигнала) от протона отдачи имитирует регистрацию позитрона. Второй (стоповый или задержанный сигнал) в обоих случаях появляется при захвате нейтрона гадолинием. Разница между этими стартовыми сигналами состоит в появлении двух гамма-квантов от аннигиляции позитрона в нейтринном процессе. Пробег протона отдачи с большой вероятностью укладывается в
Вероятность двойных стартов в зависимости от места регистрации позитрона

\begin{tabular}{c|c|c|c}
\hline $\begin{array}{c}\text { Центральная } \\
\text { секция }\end{array}$ & Боковая секция & Угловая секция & Все секции \\
\hline 0.424 & 0.294 & 0.188 & 0.300
\end{tabular}

размер одной секции. Гамма кванты с энергией $511 \mathrm{keV}$ могут быть зарегистрированы в соседних секциях.

К сожалению, при размерах секции $22.5 \times 22.5 \times$ $\times 50 \mathrm{~cm}$ приблизительно $70 \%$ стартовых сигналов от нейтринных событий также могут быть зарегистрированы в пределах одной секции. И только $\sim 30 \%$ нейтринных стартовых сигналов являются многосекционными за счет регистрации гамма-квантов в соседних секциях от аннигиляции позитрона. Конечно, фактор подавления статистики нейтринных событий в три раза является мало приемлемым, поэтому анализ результатов можно делать, используя односекционные и многосекционные события, а также их сумму. Критерием отбора нейтринных событий (нейтрино подобных событий) может служить соотношение $\sim 30$ и $\sim 70 \%$ для многосекционных и односекционных событий. Таким образом, если разностный сигнал от включения (выключения) реактора появляется в соотношении $\sim 30$ и $\sim 70 \%$ для многосекционных и односекционных стартов, то его можно интерпретировать в качестве нейтринного сигнала. Это отношение зависит от структуры детектора размера секций и их числа. Поэтому нужны расчеты методом Монте-Карло для конкретной структуры.

Рассчитывалась следующая схема детектора. Детектор состоит из 16 секций $22.5 \times 22.5 \times 50 \mathrm{~cm}$ с жестко фиксированными перегородками между ними. Перегородки из оргстекла с зеркальным отражающим покрытием служат для предотвращения выхода света за пределы секции. Материал сцинтиллятора - минеральное масло $\left(\mathrm{CH}_{2}\right)$ с добавкой $\mathrm{Gd} 1 \mathrm{~g} / 1$. Световыход сцинтиллятора $10^{4}$ фотонов на $1 \mathrm{MeV}$. В модели Монте-Карло толщина перегородки не учитывалась. Прослойка воздуха между сцинтиллятором и ФЭУ позволяет улучшить энергетическое разрешение секции, так как эффект полного внутреннего отражения от поверхности жидкого сцинтиллятора снижает телесный угол выхода света из зоны, ближней к ФЭУ, это выравнивает условия сбора света по высоте секции. Отражения фотонов от стенок зеркальные. Экспоненциальная длина пробега фотонов в сцинтилляторе составляет $4 \mathrm{~m}$. Фотоны отражаются от стенок с вероятностью 0.95. В таблице представлена вероятность регистрации двойных стартов, когда основной по энергии сигнал соответствует разным ячейкам детектора. В данном примере разыгрывался процесс с энергией позитрона $4 \mathrm{MeV}$.

Вероятность регистрации двойных стартов зависит от местоположения секции: в центре, сбоку или в углу. Для всего детектора вероятность двойных стартов в среднем 
составляет 30\%. Указанная структура распределения вероятности двойных стартов характерна именно для нейтринных событий, так как определяется процессом аннигиляции позитрона с вылетом двух гамма-квантов по $511 \mathrm{keV}$.

Прежде чем приступать к основным измерениям с новой моделью детектора, были сделаны измерения с

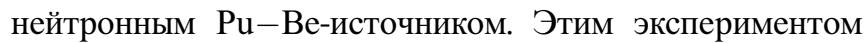
было показано, что быстрые нейтроны дают односекционные старты.

Разность скорости счета с включенным и выключенным реактором для двухсекционных и односекционных стартов, проинтегрированная по всем расстояниям, составляет $(35 \pm 7)$ и $(65 \pm 11) \%$ соответственно. В пределах имеющейся точности такое соотношение позволяет рассматривать зарегистрированные события в качестве нейтрино подобных событий. Следует также отметить и то, что многосекционная структура детектора дает возможность проводить дополнительный анализ событий, позволяя учитывать взаимное расположение стартовых и стоповых сигналов.

На рис. 2 приведены первые измерения зависимости потока антинейтрино от расстояния до активной зоны реактора (6-11 m) с прототипом секционированного нейтринного детектора.

Поскольку детектор является секционированным, зависимость от расстояния может быть представлена через каждые $0.5 \mathrm{~m}$. Метод измерений состоял в перемещении детектора на $1 \mathrm{~m}$, начиная с крайнего положения. Затем измерения повторялись со смещением начала измерений на $0.5 \mathrm{~m}$. Таким образом, обе половинки детектора измеряли одну и ту же точку, тем самым усредняя, возможно, несколько разную эффективность регистрации каждой из половинок детектора. Измерения с первым вариантом детектора без секций проводились через каждый метр, поэтому зависимость от расстояния состояла из пяти точек. С целью увеличения статистической точности измерений результаты для двух типов детекторов с зависимостью из пяти точек объединены и представлены на рис. 3.

Следует отметить, что измерения зависимости потока антинейтрино на столь близких расстояниях от активной зоны с перемещаемым детектором проведены впервые. Они представлены на рис. 4 в виде отклонения от закона $1 / R^{2}$, где $R$ - расстояние от центра активной зоны реактора до центра детектора. На этом же графике представлены результаты всех известных измерений в ближней зоне, заимствованные из работы [2]. Кроме того, для представления о масштабе возможных эффектов показаны зависимости для процесса осцилляций с четырьмя нейтрино при разных параметрах $\Delta m_{14}^{2}$ и $\sin ^{2} 2 \theta_{14}$. Из этих примеров можно заключить, что область измерений становится нечувствительна к осцилляциям для $\Delta m_{14}^{2}>2 \mathrm{eV}^{2}$. Осцилляции перемещаются в недоступную область внутри биологической защиты реактора. Для $\Delta m_{14}^{2} \approx 0.6 \mathrm{eV}^{2}$ в области измерений 6-11 m
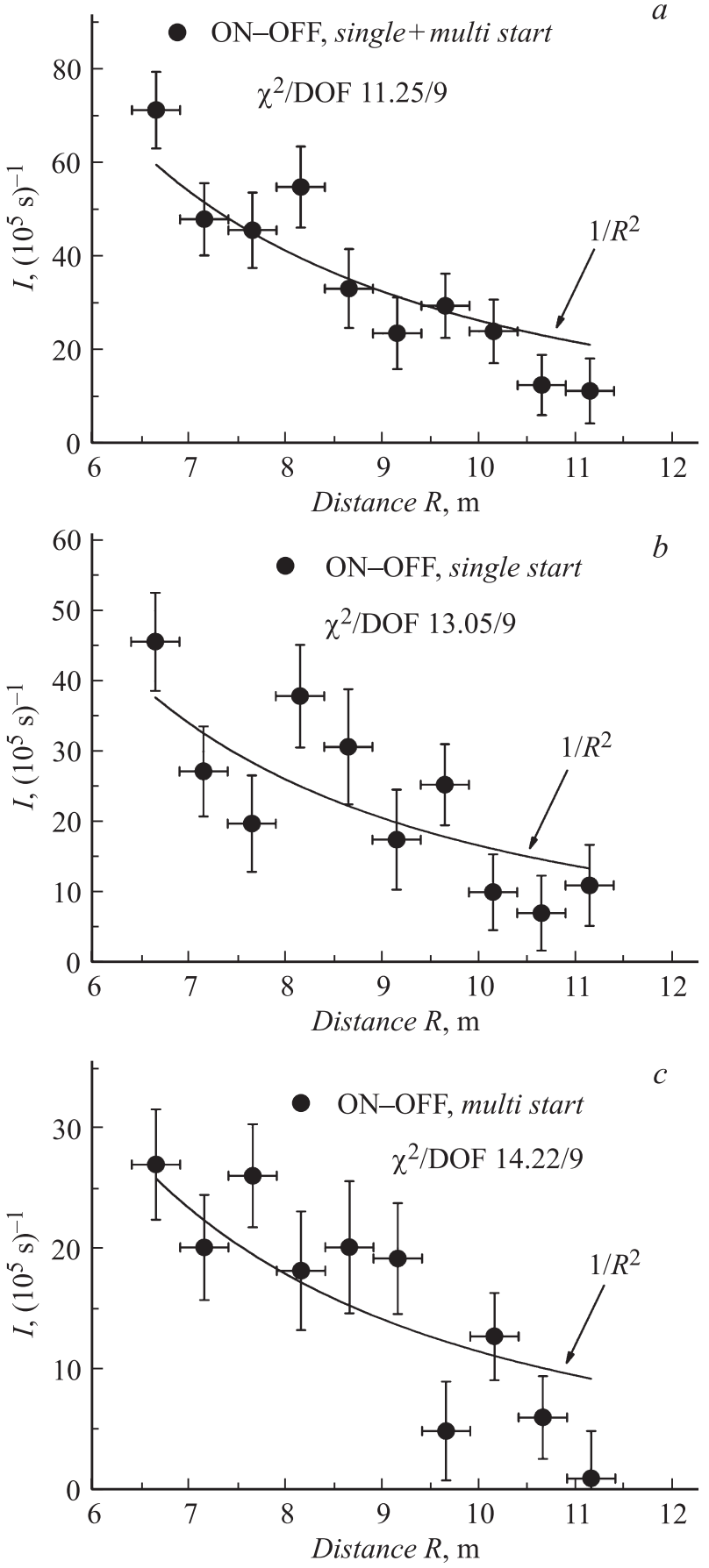

Рис. 2. Измерения зависимости потока антинейтрино от расстояния от активной зоны реактора $(6-11 \mathrm{~m})$ с секционированным прототипом нейтринного детектора. Разность скорости счета с включенным и выключенным реактором для задержанных совпадений во временном окне $100 \mu \mathrm{s}$ (за вычетом случайных совпадений) в зависимости от расстояния от центра активной зоны для трех случаев: $a-$ все события, $b$ - события с односекционными стартами, $c-$ события с многосекционными стартами.

может наблюдаться широкий минимум, и чувствительность относительных измерений с перемещаемым детектором снова пропадает. Однако в этом случае нужно 


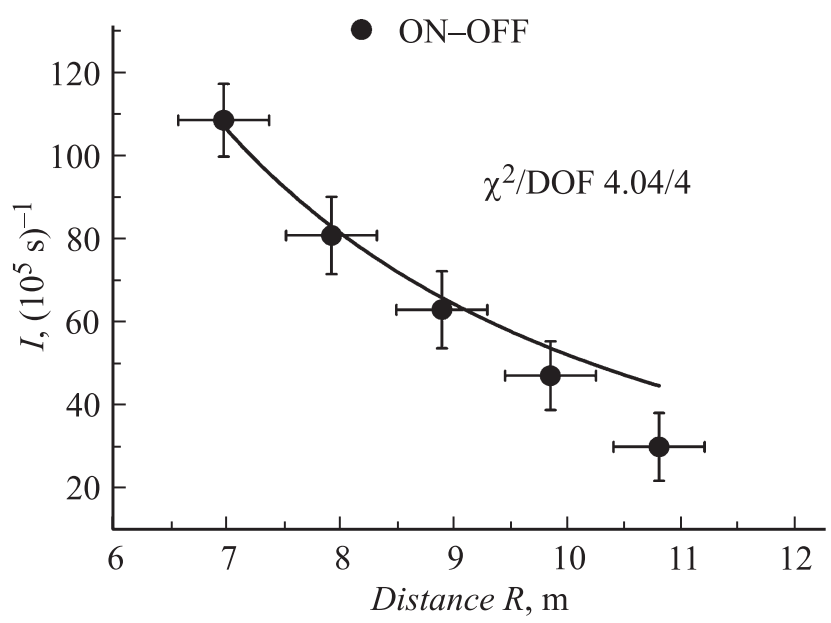

Рис. 3. Измерения зависимости потока антинейтрино от расстояния от активной зоны реактора $(6-11 \mathrm{~m})$ с обоими прототипами нейтринного детектора.

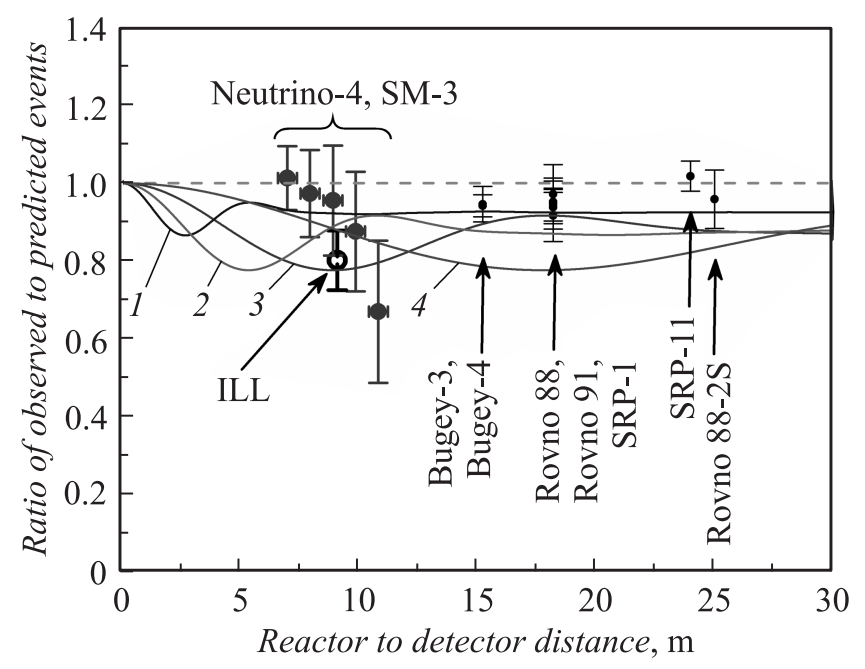

Рис. 4. Измерения зависимости потока антинейтрино от активной зоны с перемещаемым прототипом детектором в эксперименте Нейтрино-4 и другие измерения, выполненные со стационарными детекторами. 1 - кривая осцилляций в схеме с тремя нейтрино и одним стерильным нейтрино $\Delta m_{14}^{2}=2 \mathrm{eV}^{2}, \sin ^{2} 2 \theta_{14}=0.15 ; 2-\Delta m_{14}^{2}=1.0 \mathrm{eV}^{2}$, $\sin ^{2} 2 \theta_{14}=0.25 ; 3-\Delta m_{14}^{2}=0.6 \mathrm{eV}^{2}, \sin ^{2} 2 \theta_{14}=0.25 ; 4-$ $\Delta m_{14}^{2}=0.3 \mathrm{eV}^{2}, \sin ^{2} 2 \theta_{14}=0.25$.

исследовать вариацию спектра антинейтрино. Наиболее чувствительными к пространственным осцилляциям являются случаи с $\Delta m_{14}^{2} \approx 1.0 \mathrm{eV}^{2}$ и $\Delta m_{14}^{2} \approx 0.3 \mathrm{eV}^{2}$. Требуемая точность измерений для каждой точки должна составлять $\sim 2 \%$.

Среди предыдущих измерений наиболее близкой точкой к реактору является точка на расстоянии $9 \mathrm{~m}$, выполненная со стационарным детектором под реактором института Лауэ-Ланжевена (Франция) [6]. Измерения при расстоянии $15 \mathrm{~m}$ были выполнены на ядерных реакторах атомной электростанции в Буже (Франция) [7,8], измерения при расстоянии $18 \mathrm{~m}$ - на ядерном реакторе Ровенской АЭС (Украина) $[9,10]$, а также на реакторе в Саванна-Ривер (США) [11]. И наконец, измерения при расстоянии $24 \mathrm{~m}$ - на реакторе в Саванна-Ривер (США) [11] и расстоянии $25 \mathrm{~m}$ на Ровенской АЭС [9]. Все они также были выполнены со стационарными детекторами, калиброванными на измерение абсолютного нейтринного потока, и нормированы на расчетный поток антинейтрино от реактора. Детекторы располагались под толщей бетонной защиты атомных станций приблизительно $30 \mathrm{~m}$ водного эквивалента [12]. Фон от мюонов подавлялся биологической защитой реактора. Так, например, в эксперименте в Саванна-Ривер космический фон был подавлен в 4.3 раза по сравнению с фоном на поверхности Земли. В этих условиях определение эффективности детектора к регистрации антинейтрино с точностью 5-10\% представляется возможным, учитывая также то, что поток антинейтрино от атомных станций более чем на порядок величины превышает поток от реактора СМ-3. В этих условиях отношение сигнал-фон больше единицы. В нашем эксперименте изза сложных условий космического фона на поверхности Земли и отношения сигнал-фон 25-30\% претендовать на определение эффективности детектора с аналогичной точностью проблематично.

Преимущество нашего эксперимента состоит в точности относительных измерений благодаря перемещению одного и того же детектора, чтобы наблюдать возможные осцилляции. Задача состоит в обнаружении отклонения от закона $1 / R^{2}$. Тем не менее мы планируем провести подробные расчеты Монте-Карло эффективности детектора. По предварительным оценкам, расчетная эффективность секционированного детектора должна составлять 30-35\%, а экспериментальная оценка показывает приблизительно 20-25\%. Рассматривать это расхождение как подтверждение реакторной антинейтринной аномалии пока преждевременно. На рис. 4 точки зависимости потока антинейтрино от расстояния

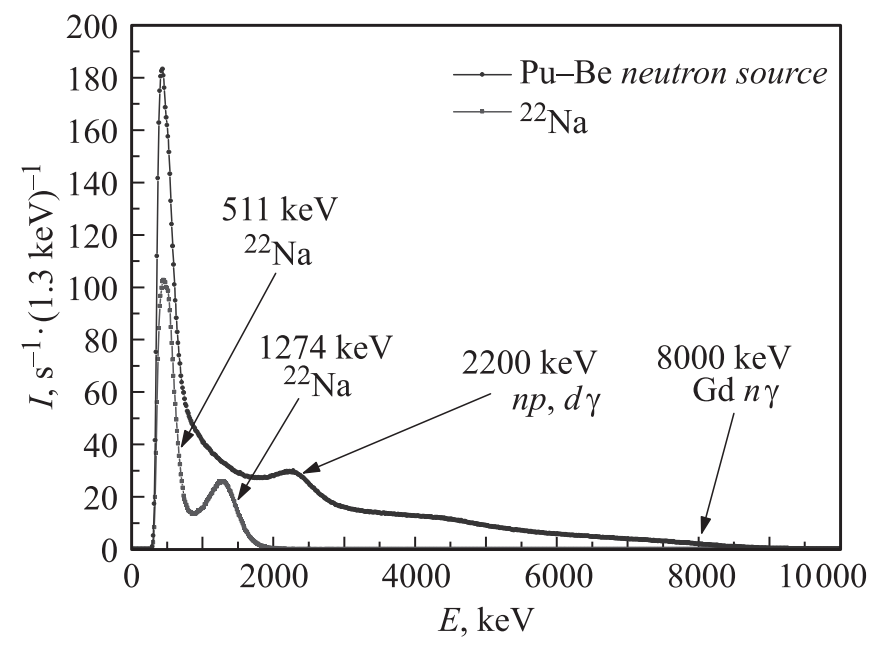

Рис. 5. Результаты энергетической калибровки детектора. 

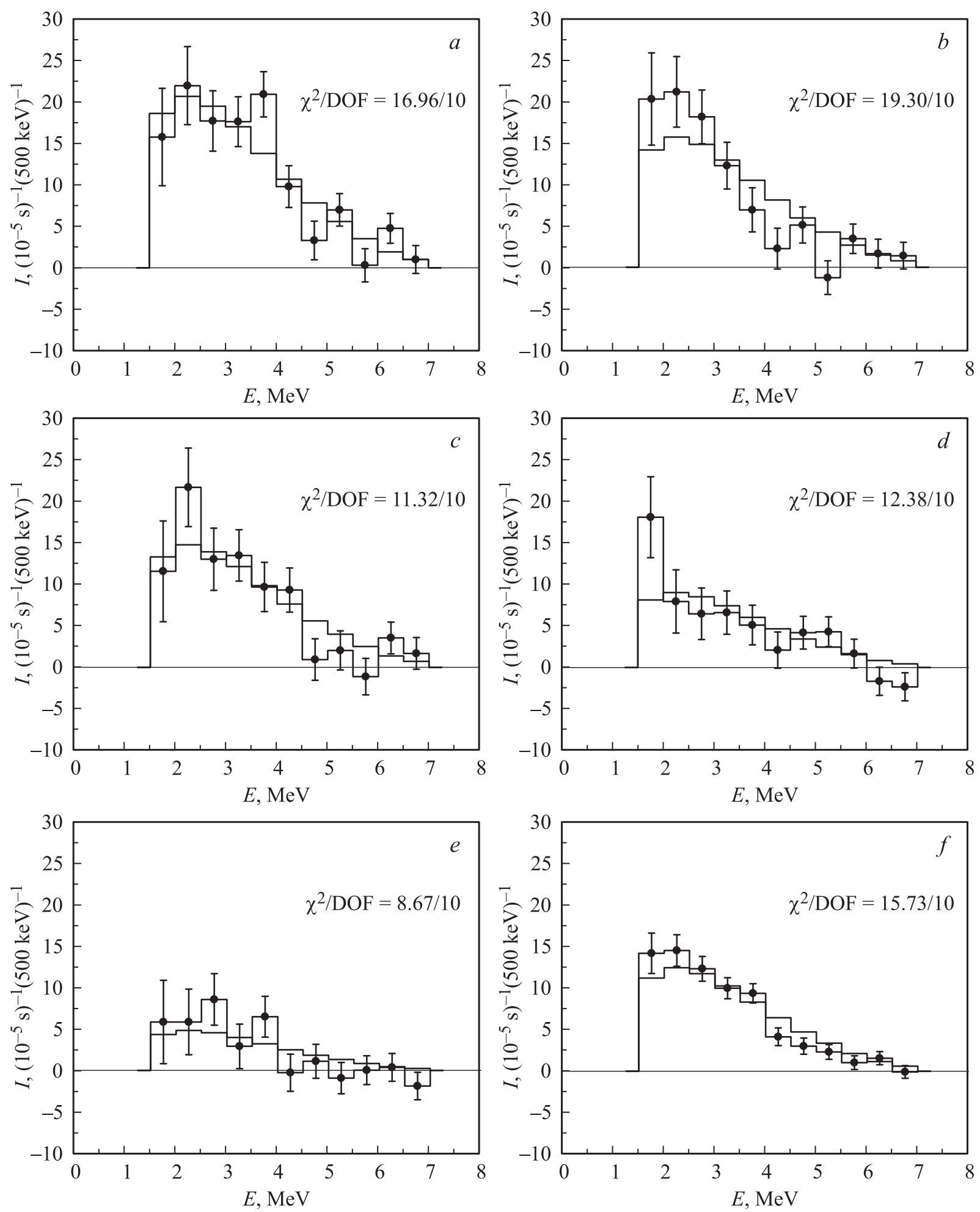

Рис. 6. Результаты измерений спектра мгновенных (позитронных) сигналов на разных расстояниях и сравнение с МК расчетами для случая без осцилляций: $a-6.9 \mathrm{~m}, b-7.9 \mathrm{~m}, c-8.9 \mathrm{~m}, d-9.9 \mathrm{~m}, e-10.9 \mathrm{~m}, f-$ средний спектр.

поставлены с предположением, что расчетная и экспериментальная эффективности регистрации детектора совпадают. Хотя надо полагать, что весь набор полученных точек на рис. 4 может быть сдвинут вниз при учете реальной эффективности детектора. При имеющейся статистической точности измерений сделать какие-либо выводы относительно осцилляций на малых расстояниях не представляется возможным.

Требуемая статистическая точность (2\%) пока не достигнута в настоящих измерениях, так как исследования 
проведены с прототипом детектора. На стадии подготовки находится полномасштабный детектор с объемом жидкого сцинтиллятора $3 \mathrm{~m}^{3}(5 \times 10$ секций). Полномасштабный детектор за два года измерений позволит добиться статистической точности в $1.5-3.0 \%$ для расстояний от 6 до $12 \mathrm{~m}$, что даст возможность внести больше ясности в вопрос о реакторной антинейтринной аномалии и возможных осцилляциях в стерильное состояние.

Как отмечалось во введении, кроме наблюдения пространственных вариаций следует исследовать вариации нейтринного спектра при перемещении детектора. Эта задача требует энергетической калибровки детектора. Перед началом эксперимента многосекционная модель детектора антинейтрино была прокалибрована с помощью источников гамма-квантов $\left({ }^{22} \mathrm{Na}\right)$ и нейтронов $(\mathrm{Pu}-\mathrm{Be})$. Результаты энергетической калибровки детектора представлены на рис. 5

На рис. 6 показаны разностные (ON-OFF) спектры мгновенных (позитронных) сигналов для пяти точек по расстоянию в сравнении с вычислениями МонтеКарло. Измеренные и вычисленные интегралы равны друг другу, чтобы наблюдать изменение формы спектра. Однако чтобы наблюдать вариацию спектров, требуется еще больше статистики, чем для наблюдения пространственных вариаций. Результаты, полученные с прототипом детектора, приводятся в данном случае в качестве иллюстрации. Можно экстраполировать, что для полномасштабного эксперимента статистические ошибки эксперимента будут уменьшены в 5-7 раз.

\section{Заключение}

Впервые выполнены измерения зависимости потока антинейтрино на близких расстояниях от активной зоны реактора с перемещаемым детектором. Измерения с прототипом многосекционного детектора показали, что в сложных условиях космического фона на поверхности Земли можно проводить измерения потока антинейтрино от реактора, но для получения необходимой точности требуется детектор значительных размеров.

Работа выполнена при поддержке Российского фонда фундаментальных исследований (грант № 14-22-03055офи_м). Настоящая работа в значительной степени сделана благодаря поставке сцинтиллятора из лаборатории под руководством профессора Jun Cao (Institute of High Energy Physics, Пекин, Китай).

\section{Список литературы}

[1] Mueller Th.A., Lhuillier D., Fallot M. et al. // Phys. Rev. C. 2011. Vol. 83. P. 054615.

[2] Mention G., Fechner M., Lasserre Th. et al. // Phys. Rev. D. 2011. Vol. 83. P. 073006.

[3] Серебров А.П., Фомин А.К., Зиновьев В.Г. и др. // Письма в ЖТФ. 2014. Т. 40. Вып. 11. С. 8.
[4] Серебров А.П., Ивочкин В.Г., Самойлов Р.М. и др. // ЖТФ. 2015. Т. 85. Вып. 12. С. 128.

[5] Серебров А.П., Ивочкин В.Г., Самойлов Р.М. и др. // ЖЭТФ. 2015. Т. 148. Вып. 4. С. 665.

[6] Kwon H., Boehm F., Hahn A.A. et al. // Phys. Rev. D. 1981. Vol. 24. P. 1097.

[7] Declais Y., de Kerret H., Lefievre B. et al. // Phys. Lett. B. 1994. Vol. 338. P. 383.

[8] Achkar B., Aleksan R., Avenier M. et al. // Nucl. Phys. B. 1994. Vol. 434. P. 503.

[9] АЯонин А.И., Кетов С.Н., Копейкин В.И. и др. // ЖЭТФ. 1988. Т. 94. С. 1.

[10] Кувшинников А.А., Микаэлян Л.А., Николаев С.В. и др. // Письма в ЖЭТФ. 1991. Т. 54. С. 259.

[11] Greenwood Z.D., Kropp W.R., Mandelkern M.A. et al. // Phys. Rev. D. 1996. Vol. 53. P. 6054.

[12] Боровой А.А., Микаэлян Л.А. // Атомная энергия. 1983. T. 54. C. 143. 